\title{
A Kuhn-Tucker model for behaviour in dictator games
}

\author{
Peter G. Moffatt ${ }^{1}$ (D) Graciela Zevallos $^{1}$
}

Received: 26 April 2020 / Revised: 31 July 2021 / Accepted: 1 November 2021 /

Published online: 3 December 2021

(c) The Author(s) 2021

\begin{abstract}
We consider a dictator game experiment in which dictators perform a sequence of giving tasks and taking tasks. The data are used to estimate the parameters of a Stone-Geary utility function over own-payoff and other's payoff. The econometric model incorporates zero observations (e.g. zero-giving or zero-taking) by applying the Kuhn-Tucker theorem and treating zeros as corner solutions in the dictator's constrained optimisation problem. The method of maximum simulated likelihood (MSL) is used for estimation. We find that selfishness is significantly lower in taking tasks than in giving tasks, and we attribute this difference to the "cold prickle of taking".
\end{abstract}

Keywords Dictator games · Taking games · Kuhn-Tucker conditions ·

Experimetrics

JEL Classification C57 · C91 · D64 · D91

\section{Introduction}

Dictator games (Forsythe et al., 1994) were originally designed with the objective of investigating the determinants of giving. Although in essence the game is very simple, a huge literature has revealed that dictator behaviour is highly sensitive to certain design features. Engel (2011) provided a thorough meta-analysis of dictator games, pooling the results of more than 100 studies. Factors he found to have a positive effect on giving include "endowment earned by recipient" and "deserving recipient". Factors having a negative effect include "endowment earned by dictator" and "repeated game".

Peter G. Moffatt

p.moffatt@uea.ac.uk

Graciela Zevallos

graciela.zevallos@uea.ac.uk

1 School of Economics and CBESS, University of East Anglia, Norwich NR4 7TJ, UK 
A particularly interesting development was the introduction of a "taking treatment". ${ }^{1}$ Bardsley (2008), List (2007) and Cappelen et al. (2013) all find evidence that the extension of the choice set to allow the dictator to take from the recipient has a negative effect on giving. Bardsley (2008) interprets this finding as the result of an experimenter demand effect (Zizzo, 2010). List's (2007) interpretation is that the social norm of the game is choice-set dependent.

A different way of investigating the impact of a taking treatment is to test for a framing effect. Here, typically the choice set is identical between the two treatments, but in one, the choice is framed as a giving decision, while in the other, it is framed as a taking decision. This sort of test has been carried out by Jakiela (2013) and Korenok et al. (2014, 2018). Common findings from these studies are that dictators tend to prefer the giving frame to the taking frame, dictators are prepared to sacrifice a proportion of their endowment to avoid the taking frame, and recipients tend to receive higher payoffs in the taking frame. In terms coined by Andreoni (1995), the "cold prickle" of taking is stronger than the "warm glow" of giving.

In this paper, we consider data from an experiment in which each dictator faces a sequence of tasks, in half of which only giving is possible, and in the remaining half only taking is possible. Between tasks, endowments and the price of transferring are varied. With this data, in a manner similar to Andreoni and Miller (2002), we estimate a parametric utility function over own-payoff and other's payoff, and the central question is whether and how the parameters of this utility function change between giving and taking tasks.

The key innovation of this paper is to develop a way of dealing consistently with observations appearing on the boundary of the feasible region (e.g. zero giving; zero taking; maximal taking). In previous studies, such observations have been treated on an equal basis to observations on the interior (Jakiela, 2013) or have been dealt with in an ad-hoc way, for example, using doubly censored regression (Andreoni \& Miller, 2002). The importance of allowing for a positive probability of zero observations and observations at other extremes (such as maximal taking), is firstly that the Nash Equilibrium prediction is typically at one of these extremes, and secondly that these observations typically make up a sizeable proportion of the experimental sample. Here, we will do more than simply allow for a positive probability of boundary observations, because we will find a way of embedding boundary observations into the theoretical model whose parameters we estimate.

The approach adopted is to apply the Kuhn-Tucker theorem (Arrow \& Enthoven, 1961) to the dictator's constrained optimisation problem, and to treat zero observations (and also observations at other extremes) as corner solutions. The resulting econometric model is similar to that of Wales and Woodland (1983), who estimated the parameters of a utility function over three highly disaggregated consumption goods using household-level data containing a high incidence of zero expenditures.

To make way for the required corner solutions, it is necessary for the dictator's utility function to be sufficiently flexible for its indifference curves to cross axes. The

\footnotetext{
1 A predecessor of the taking game was the "gangster game" introduced by Eichenberger and Oberholzer-Gee (1998), in which social norms were found to play an important role.
} 
utility function we choose with this requirement in mind is one that has not, to our knowledge, been used previously in social preference modelling: the Stone-Geary utility function. This function is best interpreted in terms of the dictator having a minimum acceptable payoff for "self" (MAPS) and a minimum acceptable payoff for "other" (MAPO). Crucially, the parameters representing MAPS and MAPO are permitted to take negative values, leading to indifference curves crossing axes, and hence to the required corner solutions. As we shall demonstrate in Sect. 3.2, it is possible to attain corner solutions at both extremes (maximal selfishness and maximal generosity) and also to attain any solution in between, simply by varying one of the parameters of the Stone-Geary utility function.

Note that the CES utility function, that has been popular in social preference modelling (Andreoni \& Miller, 2002; Jakiela, 2013) does not allow corner solutions, because the indifference curves do not cross axes. There exist other popular utility functions such as those of Fehr and Schmidt (1999) and Charness and Rabin (2002) that do allow corner solutions. However, these utility functions have the property of piecewise linear indifference curves, implying the opposite problem: interior solutions are not possible. Our Stone-Geary function satisfies strict convexity and is therefore capable of predicting any desired internal solution.

In the context of the parametric model built around the Stone-Geary assumption, we compare giving and taking treatments using what might be described as a "structural treatment test". This is a test for a change in the structural parameters between treatments. Note that this sort of test is essential in the present situation, because the effect cannot be seen by simply comparing payoffs between treatments. The test is similar to that of Jakiela (2013) who tested for a change in the CES parameters. Note that this test fulfills the key objective of the paper, which is to determine whether dictators display more or less selfishness in a taking situation than in a giving situation.

Having estimated the model, we will obtain posterior estimates of subject-specific selfishness, and use this to identify "types" in the population. We shall see that a prominent type is the "selfish type", who maximise their own payoff in every task.

The remainder of the paper is organised as follows. Section 2 describes the experimental design. Section 3 presents the theoretical model and describes the estimation procedure. Section 4 provides descriptive data analysis. Section 5 presents estimation results, with discussion thereof. Section 6 reports the results of a power analysis applied to the key test performed in Sect. 5. Section 7 concludes.

\section{Experimental design and procedure}

The experimental data used in this study was originally collected and analysed by Zevallos Porles (2018). The experiment was conducted in June 2014 at the Laboratory for Economic and Decision Research (LEDR) at the University of East Anglia. Subjects were undergraduate students, recruited through the on-line recruitment system ORSEE (Greiner, 2015). A total of 138 subjects (69 dictators and 69 recipients) participated over seven experimental sessions. Each subject participated in only one session. None of the subjects had participated in a similar experiment before. The 
Table 1 Allocation tasks

\begin{tabular}{llllllll}
\hline Task & Treatment & $\begin{array}{l}\text { Dictator's } \\
\text { endowment } \\
\left(m_{1}\right)\end{array}$ & $\begin{array}{l}\text { Recipient's } \\
\text { endowment } \\
\left(m_{2}\right)\end{array}$ & $\begin{array}{l}\text { Price of } \\
\text { self-alloca- } \\
\text { tion }\left(p_{1}\right)\end{array}$ & $\begin{array}{l}\text { Price of } \\
\text { other-allo- } \\
\text { cation }\left(p_{2}\right)\end{array}$ & $\begin{array}{l}\text { Most selfish } \\
\text { allocation } \\
\left(x_{1}, x_{2}\right)\end{array}$ & $\begin{array}{l}\text { Most gener- } \\
\text { ous allocation } \\
\left(x_{1}, x_{2}\right)\end{array}$ \\
\hline 1 & Giving & 3 & 3 & 1 & 0.5 & $(3,6)$ & $(0,12)$ \\
2 & Giving & 3 & 6 & 1 & 1 & $(3,6)$ & $(0,9)$ \\
3 & Giving & 3 & 6 & 0.5 & 1 & $(6,6)$ & $(0,12)$ \\
4 & Giving & 6 & 3 & 1 & 0.5 & $(6,6)$ & $(0,18)$ \\
5 & Giving & 6 & 6 & 1 & 1 & $(6,6)$ & $(0,12)$ \\
6 & Giving & 6 & 6 & 0.5 & 1 & $(12,6)$ & $(0,18)$ \\
7 & Giving & 10 & 3 & 1 & 0.5 & $(10,6)$ & $(0,26)$ \\
8 & Giving & 10 & 6 & 1 & 1 & $(10,6)$ & $(0,16)$ \\
9 & Giving & 10 & 6 & 0.5 & 1 & $(20,6)$ & $(0,26)$ \\
10 & Taking & 3 & 6 & 1 & 0.5 & $(9,0)$ & $(3,12)$ \\
11 & Taking & 3 & 6 & 1 & 1 & $(9,0)$ & $(3,6)$ \\
12 & Taking & 1.5 & 6 & 0.5 & 1 & $(15,0)$ & $(3,6)$ \\
13 & Taking & 6 & 6 & 1 & 0.5 & $(12,0)$ & $(6,12)$ \\
14 & Taking & 6 & 6 & 1 & 1 & $(12,0)$ & $(6,6)$ \\
15 & Taking & 3 & 6 & 0.5 & 1 & $(18,0)$ & $(6,6)$ \\
16 & Taking & 10 & 6 & 1 & 0.5 & $(16,0)$ & $(10,12)$ \\
17 & Taking & 10 & 6 & 1 & 1 & $(16,0)$ & $(10,6)$ \\
18 & Taking & 5 & 6 & 0.5 & 1 & $(22,0)$ & $(10,6)$ \\
\hline
\end{tabular}

experiment was programmed and conducted with the software z-Tree (Fischbacher, 2007).

Upon arrival, all subjects were invited into the same room and seated at one of the computer cubicles at random. In each session, subjects were given a set of printed instructions. The experimenter read the instructions aloud and answered questions. Subjects also followed the instructions on their own computer screen. All forms of communication between subjects were strictly forbidden for the whole duration of the session. After receiving the instructions, all subjects were asked to answer a set of control questions. Control questions were administered to make sure that subjects understood the experiment and these questions were computerized. Once all subjects had satisfactory answered correctly the control questions, the experiment began.

The instructions can be found in the Online Supplementary Material. The experimental design is similar to that of Andreoni and Miller (2002). In each session, dictators made allocation decisions in a series of different tasks. The tasks differ in the amount of the endowment, the price of self-allocation, the price of other-allocation, and the experimental treatment. The order of the tasks was randomized at individual level. At the start of the experiment, half of the subjects were randomly assigned to the role of dictators and the other half to the role of recipients. Dictators and recipients kept their role throughout the experiment. In each of two treatments, dictators engaged in nine allocation tasks. Table 1 below shows the dictator's endowment, the recipient's endowment and the price of transferring in each task. It also shows the most selfish and 
most generous allocations for each task. The two treatments are: "giving" (rows 1-9 of Table 1), in which dictators can give any part of their endowment in $£ 0.50$ increments; and "taking" (rows 10-18 of Table 1), in which dictators can take any part of the recipient's endowment in $£ 0.50$ increments. As seen in Table 1, the dictator's endowment was either $£ 1.5, £ 3, £ 5, £ 6$ or $£ 10$, the recipient's endowment was either $£ 3$ or $£ 6$, and the price of self-allocation and the price of other-allocation were either 1 or 0.5 .

For each task, dictators were also given a menu of possible payoff combinations associated to their decisions. Dictators made their decisions by selecting one of the payoff combinations. Once the dictator had made her decision in one of the tasks, the next task appeared on her computer screen and she was not able to go back to the previous task. Recipients had no decisions to make. Recipients were occupied and entertained by being invited to solve puzzles (unrelated to the experiment) during the time the dictators were making decisions.

Consider for example Task 4, which is a giving game. As seen in the fourth row of Table 1, the dictator's and recipient's endowments are $£ 6$ and $£ 3$ respectively, while the prices of self-allocation and other-allocation are 1 and 0.5 . If the dictator transfers zero, both players receive a payoff of $£ 6$. Note that although the recipient's endowment is only $£ 3$, her payoff is $£ 6$ because the price of other-allocation is 0.5 . If the dictator decides to transfer, say, $£ 2$ to the recipient, then the dictator's payoff reduces by $£ 2$ (to $£ 4$ ), but the recipient's payoff increases by $£ 4$ (to $£ 10$ ). The screenshot of Task 4 as presented to the dictator is shown in Figure B.1 in the Online Supplementary Material.

As a second example, consider Task 15, which is a taking game. As seen in the fifteenth row of Table 1 , the dictator's and recipient's endowments are $£ 3$ and $£ 6$ respectively, while the prices of self-allocation and other-allocation are 0.5 and 1 . Similarly to the first example, if the dictator takes zero, both players receive a payoff of $£ 6$. If the dictator decides to take, say, $£ 3$ from the recipient, then the dictator's payoff increases by $£ 6$ (to $£ 12$ ), and the recipient's payoff decreases by $£ 3$ (to $£ 3$ ). The screenshot of Task 15 as presented to the dictator is shown in Figure B.2 in the Online Supplementary Material.

After all dictators had made their decisions, they were randomly matched with one subject from the recipient group. Then, for each dictator-recipient pairing, one task was randomly chosen to determine payments. Before the payment, all subjects answered a short computerised questionnaire, which gathered data on age, gender, field of study, and country of origin.

Each experimental session lasted for about one hour and the average payment per subject (including a participation fee of $£ 2$ ) was $£ 8.59$. Payments were administered individually and privately.

\section{A model of dictator game behaviour with binding non-negativity constraints}

\subsection{Notation}

We define the following variables: 
$m_{1}=$ own endowment

$m_{2}=$ other's endowment

$x_{1}=$ amount received by self

$x_{2}=$ amount received by other

$p_{1}=$ "price" per unit of $x_{1}$ (i.e. for each unit of the endowment directed to self, self receives $1 / p_{1}$ units).

$p_{2}=$ "price" per unit of $x_{2}$ (i.e. for each unit of the endowment directed to other, other receives $1 / p_{2}$ units).

Taking products of price and "quantity", we obtain something analogous to expenditure in a consumer demand model:

$p_{1} x_{1}=$ amount directed to self

$p_{2} x_{2}=$ amount directed to other.

Note that $p_{1} x_{1}$ is the decision variable in the experiment. $p_{1} x_{1}<m_{1}$ implies positive giving; $p_{1} x_{1}>m_{1}$ implies positive taking; $p_{1} x_{1}=m_{1}$ implies zero giving or taking.

\subsection{The utility function}

With the notation set out in Sect. 3.1, we specify the Stone-Geary utility function:

$$
U\left(x_{1}, x_{2}\right)=a_{1} \ln \left(x_{1}-b_{1}\right)+a_{2} \ln \left(x_{2}-b_{2}\right) \quad x_{1}>b_{1} ; x_{2}>b_{2} .
$$

For identification of the parameters, it will be necessary to impose the normalisations:

$$
\begin{aligned}
& a_{1}+a_{2}=1 \\
& b_{1}+b_{2}=0 .
\end{aligned}
$$

In this setting, it is appropriate to interpret $b_{1}$ and $b_{2}$ as "minimum acceptable payoff for self (MAPS)" and "minimum acceptable payoff for other (MAPO)" respectively. ${ }^{2}$ Firstly, note that when $b_{1}=b_{2}=0$, (1) simplifies to the well-known Cobb-Douglas Utility function (in logarithmic form), in which the $a_{1}\left(=1-a_{2}\right)$ parameter represents selfishness. This simplification is convenient, because it allows the Cobb-Douglas model to be seen as a benchmark model. Secondly, note that an increase (or decrease) in the parameter $b_{1}$ has the effect of shifting the entire indifference map to the right (or left), while an increase (or decrease) in $b_{2}$ has the effect of shifting it up (or down). Our normalisation $b_{1}+b_{2}=0$ essentially implies that a shift of the indifference map to the right is accompanied by a downward shift of the same magnitude. This normalisation is justified partly on the grounds that variation in behaviour in the dictator game essentially amounts to movement up or down a

\footnotetext{
${ }^{2}$ When the utility function (1) is used in consumer theory, it is conventional to interpret $b_{1}$ and $b_{2}$ as "subsistence levels" of consumption of the two goods, i.e. the consumption level that is necessary for survival. See Deaton and Muellbauer (1980). This interpretation is misleading in the social preference context, and hence our preferred interpretation is in terms of minimum acceptable payoffs.
} 


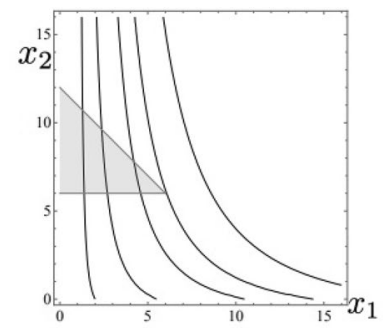

(a) Giving task: zero giving

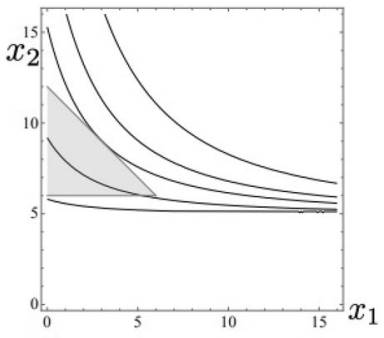

(b) Giving task: positive giving

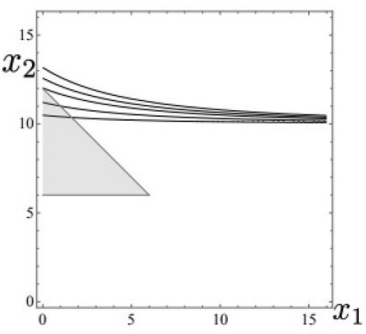

(c) Giving task: maximal giving

Fig. 1 Stone-Geary indifference maps under giving task. $x_{1}$ is own payoff; $x_{2}$ is other's payoff. Feasible region is represented by shaded triangle. In all three panels, $m_{1}=m_{2}=6 ; p_{1}=p_{2}=1$. Parameter values: a $b_{1}=1 ; a_{1}=2 / 3$; corner solution (zero giving); b $b_{1}=-5 ; a_{1}=2 / 3$; interior solution (positive giving); $\mathbf{c} b_{1}=-10 ; a_{1}=2 / 3$; corner solution (maximal giving)

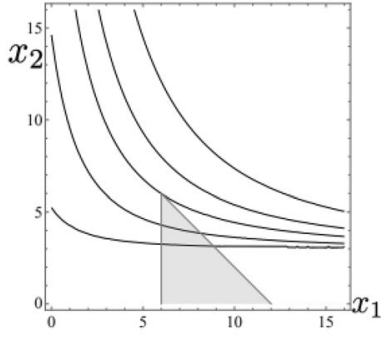

(a) Taking task: zero taking

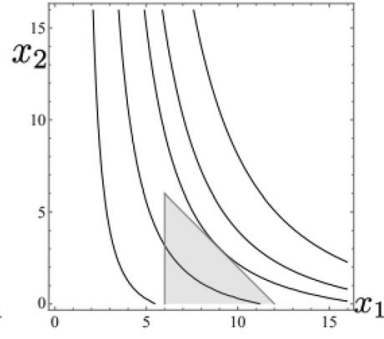

(b) Taking task: positive taking

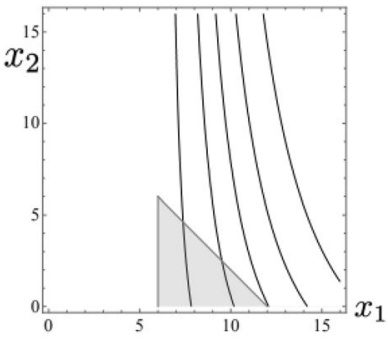

(c) Taking task: maximal taking

Fig. 2 Stone-Geary indifference maps under taking task. $x_{1}$ is own payoff; $x_{2}$ is other's payoff. Feasible region is represented by shaded triangle. In all three panels, $m_{1}=m_{2}=6 ; p_{1}=p_{2}=1$. Parameter values: a $b_{1}=-3 ; a_{1}=2 / 3$; corner solution (zero taking); b $b_{1}=1 ; a_{1}=2 / 3$; interior solution (positive taking); $b_{1}=6 ; a_{1}=2 / 3$; corner solution (maximal taking)

downward-sloping budget line; to explain such movement in terms of changes in $b_{1}$ and $b_{2}$, the two parameters must be moving in opposite directions.

The normalisation $b_{1}+b_{2}=0$ clearly implies that one of MAPS and MAPO is negative (unless they are both zero). A negative value for one of these parameters implies that indifference curves cross one of the two axes, and note that this is exactly what is required in order explain zero observations on $x_{1}$ or $x_{2}$. Specifically, we expect the MAPO, $b_{2}$, to be negative, since this makes way for zero observations in other's payoff, which is a standard feature of Dictator game data.

The assumption that the MAPS, $b_{1}$, is positive is also a natural one. It captures the plausible idea that the dictator approaches a task with a minimum acceptable payoff for themselves in mind, and only when that minimum payoff is attained 
will they consider giving to others. The proportion of "supernumerary endowment" 3 they allocate to the other player is in fact given by the parameter $a_{2}$.

Various Stone-Geary indifference maps are illustrated in Figs. 1 and 2. In each case, the feasible region is represented by the shaded triangle. ${ }^{4}$ As illustrated in the Figures, whether the solution is an interior or a corner solution depends on the positions of the indifference curves relative to the feasible region.

Figures 1 and 2 clearly demonstrate the benefits from assuming variation in the $b$ parameters. In both cases it is demonstrated that it is possible to move all the way from the most selfish outcome to the most generous outcome, and also to attain any desired outcome in between, by varying the parameter $b_{1}$ (and therefore $b_{2}$ ) and nothing else.

\subsection{Giving task-theory}

The budget constraint (in both giving and taking tasks) is:

$$
p_{1} x_{1}+p_{2} x_{2} \leqslant m_{1}+m_{2} \text {. }
$$

In the giving task, the non-negativity constraints are embodied in $0 \leqslant p_{1} x_{1} \leqslant m_{1}$. The two constraints taken together give rise to a feasible region such as those represented by the shaded triangles in Figs. 1a-c above.

The dictator's constrained optimisation problem for the giving task may be stated as:

$$
\max _{x_{1}, x_{2}} U\left(x_{1}, x_{2}\right) \quad \text { s.t. } \quad p_{1} x_{1}+p_{2} x_{2} \leqslant m_{1}+m_{2} ; \quad 0 \leqslant p_{1} x_{1} \leqslant m_{1}
$$

where $U\left(x_{1}, x_{2}\right)$ is specified in (1). The Lagrangean function is:

$$
\begin{aligned}
L= & a_{1} \ln \left(x_{1}-b_{1}\right)+a_{2} \ln \left(x_{2}-b_{2}\right)+\lambda\left(m_{1}+m_{2}-p_{1} x_{1}-p_{2} x_{2}\right) \\
& +\mu_{1} p_{1} x_{1}+\mu_{2}\left(m_{1}-p_{1} x_{1}\right) .
\end{aligned}
$$

Applying the Kuhn-Tucker theorem (Arrow \& Enthoven, 1961) to (5), we obtain the following complementary slackness conditions:

\footnotetext{
${ }^{3}$ In demand analysis, "supernumerary income" is the term used for the portion of a consumer's income that remains after all of the consumer's basic needs have been met. See Deaton and Muellbauer (1980).

${ }^{4}$ Strictly speaking the feasible region is a single line-the hypotenuse of the shaded triangle. This is because the rules of the game stipulate that the total endowment must be divided between dictator and recipient. However, given the assumption of an increasing utility function, the implications of the model are the same if we present the feasible region as a triangle. This is convenient since it brings the analysis into alignment with standard textbook treatment of the 2-good model in consumer theory.
} 


$$
\begin{aligned}
& \frac{a_{1}}{p_{1}\left(x_{1}-b_{1}\right)}<\frac{a_{2}}{p_{2}\left(x_{2}-b_{2}\right)} \quad \Leftrightarrow \quad p_{1} x_{1}=0 \\
& \frac{a_{1}}{p_{1}\left(x_{1}-b_{1}\right)}=\frac{a_{2}}{p_{2}\left(x_{2}-b_{2}\right)} \quad \Leftrightarrow \quad 0<p_{1} x_{1}<m_{1} \\
& \frac{a_{1}}{p_{1}\left(x_{1}-b_{1}\right)}>\frac{a_{2}}{p_{2}\left(x_{2}-b_{2}\right)} \quad \Leftrightarrow \quad p_{1} x_{1}=m_{1} .
\end{aligned}
$$

Let us focus on the self-allocation $\left(x_{1}\right)$. Combining the complementary slackness conditions (6) with the (binding) budget constraint $\left(p_{1} x_{1}+p_{2} x_{2}=m_{1}+m_{2}\right)$, we obtain the well-known linear expenditure system (with non-negativity constraints):

$$
\begin{aligned}
& p_{1} x_{1}=p_{1} b_{1}+a_{1}\left(m_{1}+m_{2}-p_{1} b_{1}-p_{2} b_{2}\right) \quad \Leftrightarrow \quad 0<\text { RHS }<m_{1} \\
& p_{1} x_{1}=0 \quad \Leftrightarrow \quad \text { RHS } \leqslant 0 \\
& p_{1} x_{1}=m_{1} \quad \Leftrightarrow \quad \text { RHS } \geqslant m_{1} .
\end{aligned}
$$

In (7), "RHS" denotes the right-hand side of the first equation. The first equation of (7) has the following interpretation. Given an interior solution, expenditure on good $1\left(p_{1} x_{1}\right)$ is the expenditure required to attain the minimum acceptable payoff for self $\left(p_{1} b_{1}\right)$ plus a proportion $a_{1}$ of supernumerary endowment.

Applying the normalisations (2), and after some rearranging, the three conditions (7) may be written:

$$
\begin{aligned}
& b_{1}<\frac{-a_{1}\left(m_{1}+m_{2}\right)}{\left(1-a_{1}\right) p_{1}+a_{1} p_{2}} \Leftrightarrow p_{1} x_{1}=0 \\
& b_{1}=\frac{p_{1} x_{1}-a_{1}\left(m_{1}+m_{2}\right)}{\left(1-a_{1}\right) p_{1}+a_{1} p_{2}} \Leftrightarrow 0<p_{1} x_{1}<m_{1} \\
& b_{1}>\frac{m_{1}-a_{1}\left(m_{1}+m_{2}\right)}{\left(1-a_{1}\right) p_{1}+a_{1} p_{2}} \Leftrightarrow \quad p_{1} x_{1}=m_{1} .
\end{aligned}
$$

\subsection{Taking task-theory}

In the taking task, the non-negativity constraints are represented by $m_{1} \leqslant p_{1} x_{1} \leqslant m_{1}+m_{2}$. The lower bound of this double inequality represents zero taking, while the upper bound represents maximal taking. This constraint, together with the budget constraint (3), gives rise to a feasible region such as those represented by the shaded triangles in Fig. $2 \mathrm{a}-\mathrm{c}$ above.

The constrained optimisation problem may be stated as:

$$
\max _{x_{1}, x_{2}} U\left(x_{1}, x_{2}\right) \quad \text { s.t. } \quad p_{1} x_{1}+p_{2} x_{2} \leqslant m_{1}+m_{2} ; \quad m_{1} \leqslant p_{1} x_{1} \leqslant m_{1}+m_{2} .
$$

As with the giving task considered in Sect. 3.3, we apply a version of the Kuhn-Tucker theorem, and arrive at a set of three conditions analogous to (8) above: 
Table 2 Definitions of the three behavioural regimes for each type of task

\begin{tabular}{lll}
\hline Regime & Giving tasks & Taking tasks \\
\hline I & $p_{1} x_{1}=0$ & $p_{1} x_{1}=m_{1}$ \\
II & $0<p_{1} x_{1}<m_{1}$ & $m_{1}<p_{1} x_{1}<m_{1}+m_{2}$ \\
III & $p_{1} x_{1}=m_{1}$ & $p_{1} x_{1}=m_{1}+m_{2}$ \\
\hline
\end{tabular}

$$
\begin{aligned}
& b_{1}<\frac{m_{1}-a_{1}\left(m_{1}+m_{2}\right)}{\left(1-a_{1}\right) p_{1}+a_{1} p_{2}} \Leftrightarrow p_{1} x_{1}=m_{1} \\
& b_{1}=\frac{p_{1} x_{1}-a_{1}\left(m_{1}+m_{2}\right)}{\left(1-a_{1}\right) p_{1}+a_{1} p_{2}} \Leftrightarrow m_{1}<p_{1} x_{1}<m_{1}+m_{2} \\
& b_{1}>\frac{\left(1-a_{1}\right)\left(m_{1}+m_{2}\right)}{\left(1-a_{1}\right) p_{1}+a_{1} p_{2}} \Leftrightarrow p_{1} x_{1}=m_{1}+m_{2} .
\end{aligned}
$$

\subsection{Likelihood function}

Whichever of the two types of task (giving or taking) is being considered, the dictator's decision falls into one of three "regimes" of behaviour: lower bound (regime I); interior solution (regime II); upper bound (regime III). Since the three regimes are defined differently in giving and taking tasks, we provide explicit definitions in Table 2.

Let $i=1, \ldots, n$ index dictators, and let $t=1, \ldots, T$ index tasks. In order to allow within-subject preference variability, and also to allow a treatment effect, we shall assume that the minimum acceptable payoff for self (MAPS), $b_{1}$, varies between tasks. Denote the value of this parameter for dictator $i$ in task $t$ as $b_{1, i t}$. We will assume:

$$
b_{1, i t} \sim N\left(\gamma_{i}+\beta_{\mathrm{take}} d_{\mathrm{take}, t}+\beta_{\tau} \tau_{i t}, \sigma^{2}\right)
$$

where $\gamma_{i}$ is the baseline mean MAPS for dictator $i, d_{\text {take, } t}$ is a treatment dummy indicating whether (one) or not (zero) task $t$ is a taking task, and $\tau_{i t}$ is the position of task $t$ in the ordering of tasks faced by dictator $i$. The key parameter of the model is $\beta_{\text {take }}$, since this represents the treatment effect of central interest. The parameter $\beta_{\tau}$ represents the change in selfishness with experience of the task.

To construct the likelihood function, it is convenient to define the quantity $z_{i t}$ as:

$$
z_{i t}=\frac{\frac{p_{1, t} x_{1, i t}-a_{1}\left(m_{1, t}+m_{2, t}\right)}{\left(1-a_{1}\right) p_{1, t}+a_{1} p_{2, t}}-\left(\gamma_{i}+\beta_{\mathrm{take}} d_{\mathrm{take}, t}+\beta_{\tau} \tau_{i t}\right)}{\sigma} .
$$

Conditional on the (baseline) subject mean $\gamma_{i}$, the likelihood contributions associated with a single observation in each of the three regimes (defined in Table 2) are then: 


$$
\begin{aligned}
& \text { I: } L_{i t}^{I} \mid \gamma_{i}=\Phi\left[z_{i t}\right] \\
& \text { II: } L_{i t}^{I I} \mid \gamma_{i}=\frac{1}{\sigma} \phi\left[z_{i t}\right] \\
& \text { III: } L_{i t}^{I I I} \mid \gamma_{i}=1-\Phi\left[z_{i t}\right]
\end{aligned}
$$

where $\phi($.$) and \Phi($.$) are the standard normal p.d.f. and c.d.f. respectively, and z_{i t}$ is defined in (12). Note that the likelihood contributions defined in (13) apply to both giving and taking tasks.

If we then define three indicators $D_{i t}^{\mathrm{I}}, D_{i t}^{\mathrm{II}}, D_{i t}^{\mathrm{III}}$ taking the value 1 if subject $\mathrm{i}$ in task $\mathrm{t}$ is in regime I, II, and III respectively, and zero otherwise, we can express the conditional likelihood contribution for this single observation as:

$$
L_{i t} \mid \gamma_{i}=\left(L_{i t}^{\mathrm{I}} \mid \gamma_{i}\right)^{D_{i t}^{\mathrm{I}}}\left(L_{i t}^{\mathrm{II}} \mid \gamma_{i}\right)^{D_{i t}^{\mathrm{II}}}\left(L_{i t}^{\mathrm{III}} \mid \gamma_{i}\right)^{D_{i t}^{\mathrm{II}}} .
$$

The likelihood contribution (still conditional on $\gamma_{i}$ ) for subject $i$ is then:

$$
L_{i} \mid \gamma_{i}=\prod_{t=1}^{T}\left(L_{i t} \mid \gamma_{i}\right)
$$

Between-subject heterogeneity may be introduced by allowing the (baseline) mean parameter $\gamma_{i}$ to vary between subjects. Accordingly, we assume:

$$
\gamma_{i} \sim N\left(\mu, \eta^{2}\right)
$$

The marginal likelihood contribution for subject $\mathrm{i}$ is obtained by integrating (15) over $\gamma$, as follows:

$$
L_{i}\left(a_{1}, \mu, \beta_{\text {take }}, \beta_{\tau}, \eta, \sigma\right)=\int_{-\infty}^{\infty}\left[\prod_{t=1}^{T}\left(L_{i t} \mid \gamma\right)\right] \frac{1}{\eta} \phi\left(\frac{\gamma-\mu}{\eta}\right) \mathrm{d} \gamma .
$$

Finally, the sample log-likelihood function may be written as:

$$
\log L\left(a_{1}, \mu, \beta_{\mathrm{take}}, \beta_{\tau}, \eta, \sigma\right)=\sum_{i=1}^{n} \ln \left(L_{i}\left(a_{1}, \mu, \beta_{\mathrm{take}}, \beta_{\tau}, \eta, \sigma\right)\right) .
$$

The sample log-likelihood (18) is maximised with respect to the model's six free parameters, $a_{1}, \mu, \beta_{\text {take }}, \beta_{\tau}, \eta, \sigma$, to obtain estimates thereof. ${ }^{5}$

\footnotetext{
5 The method of maximum simulated likelihood (MSL; Train, 2009) is used, with the integral in (17) being evaluated using Halton draws. The program is written in STATA 16 and is available on request. See Moffatt (2015) for detailed accounts of the use of MSL in settings similar to this.
} 
Table 3 Distribution of decisions between the three behavioural regimes, separately for giving tasks and taking tasks

\begin{tabular}{llllll}
\hline Regime & Definition & Giving tasks & $\begin{array}{l}\text { Proportion of giv- } \\
\text { ing tasks }(\%)\end{array}$ & Taking tasks & $\begin{array}{l}\text { Proportion of } \\
\text { taking tasks } \\
(\%)\end{array}$ \\
\hline I & Lower bound & 1 & 0.2 & 75 & 12.1 \\
II & Interior solution & 74 & 11.9 & 199 & 32.1 \\
III & Upper bound & 546 & 87.9 & 347 & 55.9 \\
\hline
\end{tabular}

Total number of giving tasks: 621 . Total number of taking tasks: 621

\subsection{Posterior estimates of selfishness parameter}

Having estimated the model, it is useful to obtain posterior estimates of the mean MAPS for each subject. Such posterior estimates are obtained using Bayes' Rule:

$$
\widehat{\gamma}_{i}=\frac{\int_{-\infty}^{\infty} \gamma\left[\prod_{t=1}^{T}\left(\hat{L}_{i t} \mid \gamma\right)\right] \frac{1}{\hat{\eta}} \phi\left(\frac{\gamma-\hat{\mu}}{\hat{\eta}}\right) \mathrm{d} \gamma}{\int_{-\infty}^{\infty}\left[\prod_{t=1}^{T}\left(\widehat{L}_{i t} \mid \gamma\right)\right] \frac{1}{\hat{\eta}} \phi\left(\frac{\gamma-\hat{\mu}}{\hat{\eta}}\right) \mathrm{d} \gamma} .
$$

In (19), hats indicate that parameters have been replaced by estimates.

\section{Data}

Data from the experiment described in Sect. 2 have been used to estimate the model developed in Sect. 3.

There are 69 subjects carrying out 18 tasks, of which 9 are giving tasks and 9 are taking tasks. As seen in Table 1 above, the tasks differ in the amount of the endowment, the price of self-allocation, and the price of other-allocation. To give a feel for the data, Table 3 shows the distribution of the data between the three behavioural regimes defined in Table 2 above. We see that in giving tasks there is a strong bias towards selfishness with almost $90 \%$ of giving decisions being zero. In taking tasks, while there is again a bias towards the most selfish regime, the distribution of taking decisions between the three regimes is much more even. The high numbers in the final row of Table 3 (implying maximal selfishness) underline the importance of allowing for corner solutions in the theoretical model.

Further descriptive analysis of the data is provided in Fig. 3, where we provide jittered scatter plots of amount received by other, against amount received by self, separately for the giving tasks and the taking tasks. Firstly, note that in both plots, as expected, observations are grouped on downward-sloping straight lines, corresponding to the budget lines implied by the endowment-price combinations in the design (see Table 1). 

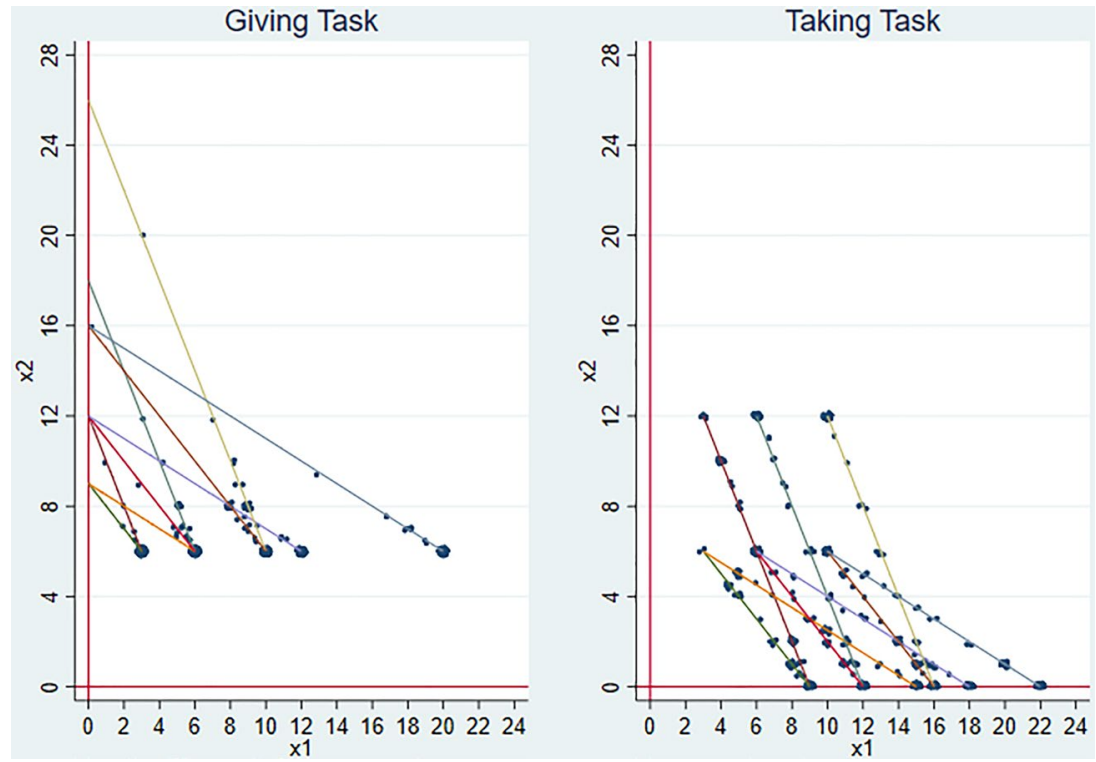

Fig. 3 Jittered scatterplots of amount received by other $\left(x_{2}\right)$, against amount received by self $\left(x_{1}\right)$, separately for giving tasks (left panel) and taking tasks (right panel). Each straight line represents the set of feasible allocations for one of the tasks

Table 4 Maximum likelihood estimates

\begin{tabular}{lll}
\hline Parameter & All subjects & Selfish subjects excluded \\
\hline$a_{1}$ & $0.550(0.036)$ & $0.550(0.035)$ \\
$\mu$ & $10.158(1.083)$ & $6.176(0.843)$ \\
$\beta_{\text {take }}$ & $-1.648(0.411)$ & $-1.586(0.404)$ \\
$\beta_{\tau}$ & $-0.048(0.031)$ & $-0.052(0.031)$ \\
$\eta$ & $6.484(0.677)$ & $3.558(0.373)$ \\
$\sigma$ & $3.506(0.167)$ & $3.481(0.165)$ \\
$\log L$ & -1049.36 & -999.734 \\
$n$ & 69 & 47 \\
$T$ & 18 & 18
\end{tabular}

Estimated model is defined in (1), (11), (16). Estimation is by maximum simulated likelihood. Asymptotic standard errors in parentheses

Secondly note that, in both graphs, a high proportion of observations lie on a lower bound of $x_{2}$, implying either zero giving or maximal taking. This accords with the high numbers appearing in the final row of Table 3, interpreted above.

Finally, note that a comparison of the two plots indicates that dictator behaviour appears to be more selfish in taking tasks than in giving tasks. To confirm this, the mean of dictator's payoff is higher in the taking treatment (£12.34) than in the 


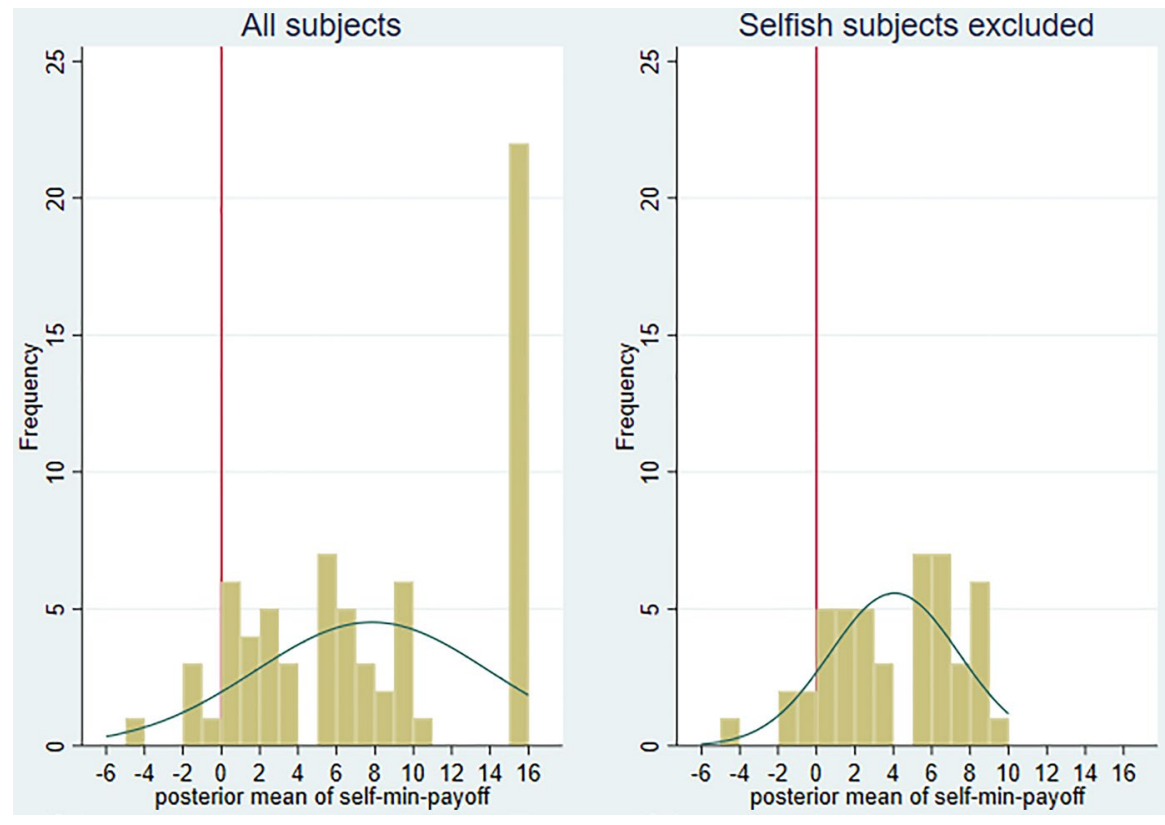

Fig. 4 The distribution of posterior mean of minimum acceptable payoff for self (MAPS) for the two estimations. Normal densities superimposed

giving treatment (£8.25), while the mean of recipient's payoff is lower in the taking treatment (£2.28) than in the giving treatment (£6.24). However, these comparisons are misleading, since it is a feature of our design that taking tasks are-regardless of dictator preferences - prone to higher payoffs to the dictator and lower payoffs to the recipient (than in giving tasks). This is essentially because for given endowments, a taking task is at least as favorable to the dictator as a giving task. To identify the true underlying effect of the taking treatment on selfishness, it is necessary to conduct a structural treatment test of the type performed in the next section.

\section{Results}

The results from estimating the model constructed in Sect. 3 using the data described in Sect. 4 are presented in Table 4 .

The first column of Table 4 presents results from estimation with the complete sample. The key result is the strong negative significance of the estimate of the treatment effect $\beta_{\text {take }}$, indicating that there is strong evidence that selfishness is lower in taking tasks than in giving tasks, ceteris paribus. The precise interpretation of the estimate of $\beta_{\text {take }}$ is that dictators are prepared to settle for a payoff of $£ 1.65$ less when the task is a taking task, presumably in an attempt to allay the "cold prickle" of guilt associated with the task. 
Posterior means $\left(\hat{\gamma}_{i}\right)$ of the MAPS for each subject (applying to giving tasks) are obtained using (19) above, and their distribution is shown in the left panel of Fig. $4{ }^{6}$ The striking feature of this histogram is the prominent cluster of subjects at the right-hand end of the distribution. It turns out that this cluster consists of the 22 (out of 69) subjects who exhibit maximal selfishness in every task; that is, they give zero in every giving task, and they take the recipient's entire endowment in every taking task. We label such subjects as "selfish". ${ }^{7}$ The presence of this cluster calls into question the assumption (16) of Normality of $\gamma_{i}$.

As a robustness check, we re-estimate the model excluding the 22 selfish subjects. The results are shown in the second column of Table 4 . The key result remains the same: the estimate of $\beta_{\text {take }}$ is significantly negative, confirming that selfishness is significantly lower in taking tasks than in giving tasks. The posterior means from estimation with the restricted sample are shown in the second panel of Fig. 4. As expected, the right-hand cluster has disappeared, and the remainder of the distribution has become more compact, in agreement with the much lower estimate of $\eta$. Moreover, the distribution appears closer to normality.

Note that the model with selfish subjects excluded is essentially equivalent to a finite mixture model with two types: selfish and non-selfish. The mixing proportion for the selfish type is estimated as the proportion of the original sample who have been classified as selfish using Fig. 4 , that is, 22/69 $=0.32$.

The other selfishness parameter is $a_{1}$, representing the proportion of supernumerary endowment allocated to self. In both estimations, this parameter is not significantly different from 0.5 . This leads to the interesting conclusion that once dictators have satisfied their self-determined basic requirement, they are happy to divide the remainder of the endowment equally between themselves and the recipient.

Finally, we see that in both estimations the estimate of $\beta_{\tau}$ is negative but not significant. This is consistent with the stability of preferences over the course of the experiment.

\section{Power analysis}

Here we report on a Monte Carlo exercise which is used to compute the power of the central treatment test of Sect. 5, that is, the test of $H_{0}: \beta_{\text {take }}=0$ against $H_{1}: \beta_{\text {take }}<0$. The Monte Carlo approach is essential here because the test has been conducted in an estimation framework that is non-standard.

The data generating process (DGP) consists of equations (8), (10), (11) and (16), with parameters replaced by estimates from the first column of Table 4. The experimental design is the same as the one used in the real experiment, as shown in Table 1. 10,000 replications were used for each run. For each run, the proportion of replications for which the null hypothesis was rejected (i.e. for which $p$-value

\footnotetext{
${ }^{6}$ The distribution for taking tasks is the same but shifted to the left by the estimate of $\beta_{\text {take. }}$.

${ }^{7}$ It is possible that this is an over-estimate of the number of "truly selfish" subjects, since there are no tasks in the design that distinguish truly selfish subjects from those who are very nearly so.
} 


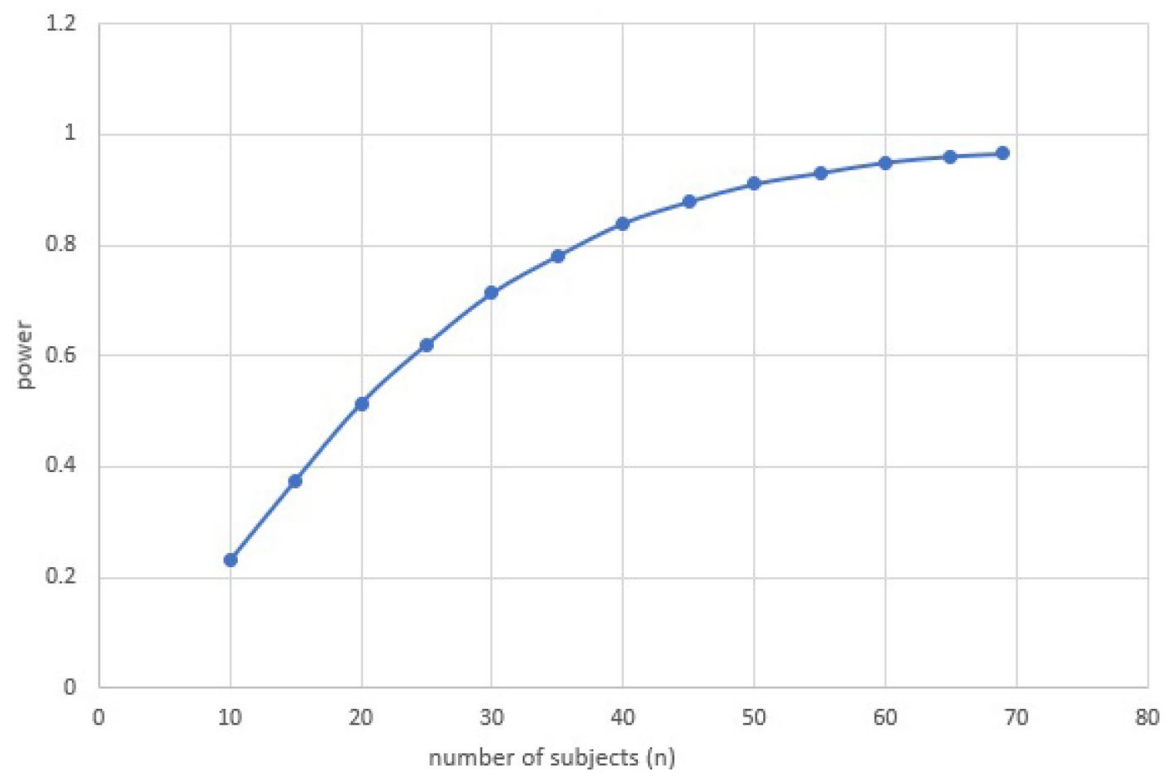

Fig. 5 Results from Monte Carlo experiment. Power (proportion of 10,000 replications for which null is rejected) for various values of $n$ (number of subjects). Simulated subjects each engage in the 18 tasks presented in Table 1. DGP consists of Eqs. (8), (10), (11), and (16) with parameter values set to the estimates reported in first column of Table 4

$<0.05)$ was recorded and provides an estimate of the power of the test. The only quantity that is altered between runs is the number of subjects $(n)$.

The results are presented graphically in Fig. $5 .^{8}$ We see that the ex-post power of the test performed in Sect. 5 (with $n=69$ ) is 0.965 . This power is considerably higher than the benchmark power of 0.80 that many experimentalists set as a target. From the graph, we also see that the benchmark power of 0.80 could be achieved with the much smaller number of subjects, 37 .

For good measure we also compute the actual test size. This is obtained by setting the value of $\beta_{\text {take }}$ to zero in the DGP, and finding the proportion of replications for which $H_{0}: \beta_{\text {take }}=0$ is incorrectly rejected in favour of $H_{1}: \beta_{\text {take }}<0$. Again with 10,000 replications, we find that the actual test size is very close to (and not significantly different from) the nominal test size of 0.05 . This implies that the test is unbiased.

Overall, results from the power analysis may be taken to infer that the experimental design is fit for purpose, and that the chosen sample size of 69 (dictators) is more than adequate.

\footnotetext{
${ }^{8}$ The Monte Carlo experiment was run using the Simulate command in STATA. The STATA code is available from the authors on request.
} 


\section{Conclusion}

We have contributed to the literature on taking dictator games initiated by Bardsley (2008) and List (2007). In the experimental setting that we have considered, the theoretical (Nash) prediction is either zero giving or maximal taking, and there is a high incidence of such observations in the data. For these reasons, it is clearly very important for any theoretical model to assign positive probability to these observations. We have accomplished this by treating zero giving and maximal taking as corner solutions in the dictator's constrained optimisation problem.

The Stone-Geary utility function has been assumed. This is mainly because this utility function has indifference curves crossing axes, which makes way for the required corner solutions. The Stone-Geary assumption also leads to a novel characterisation of selfishness, in which the dictator may be thought of as first deciding (privately) how much of an endowment is rightfully theirs (referred to as the "minimum acceptable payoff for self", or MAPS), and then deciding the proportion of the remaining endowment they are prepared to share with the recipient. As expected, post-estimation analysis reveals wide between-subject variation in MAPS. In particular, there is a clearly identified cluster of dictators with a very high MAPS and these are, of course, the dictators who exhibit fully selfish behaviour in every task. This sort of heterogeneity usually calls for the use of a finite mixture model (see Moffatt, 2015). In the present case, estimation of the mixture model is accomplished simply by estimating the model excluding subjects identified as selfish. The proportion of selfish subjects has been estimated at around one third.

We conducted a power analysis to confirm the validity of the hypothesis test of central interest. At the same time the power analysis was used to confirm the appropriateness of the experimental design.

The most important conclusion from the analysis is that selfishness is significantly lower in taking tasks than in giving tasks, ceteris paribus. Note that the structural treatment test was essential in arriving at this finding. As discussed at the end of Sect. 4, the result cannot be obtained by simply comparing payoffs between treatments, and this provides a strong justification for the fully structural approach adopted in this paper. The result provides fresh evidence that the "cold prickle of taking" is an important influence on agents' behaviour in social preference settings.

Perhaps surprisingly, the second selfishness parameter $\left(a_{1}\right)$ is estimated as being very close to 0.5 , indicating that dictators behave like egalitarians once their minimum acceptable payoff has been realised.

Supplementary Information The online version contains supplementary material available at https://doi. org/10.1007/s40881-021-00110-y.

Acknowledgements We are grateful to the Editors and two anonymous reviewers for their detailed and insightful feedback that has led to significant improvements in the paper. We are also grateful to participants at the 2020 ESA Global Online Around-the-Clock Conference for useful comments. 
Open Access This article is licensed under a Creative Commons Attribution 4.0 International License, which permits use, sharing, adaptation, distribution and reproduction in any medium or format, as long as you give appropriate credit to the original author(s) and the source, provide a link to the Creative Commons licence, and indicate if changes were made. The images or other third party material in this article are included in the article's Creative Commons licence, unless indicated otherwise in a credit line to the material. If material is not included in the article's Creative Commons licence and your intended use is not permitted by statutory regulation or exceeds the permitted use, you will need to obtain permission directly from the copyright holder. To view a copy of this licence, visit http://creativecommons.org/licen ses/by/4.0/.

\section{References}

Andreoni, J. (1995). Warm-glow versus cold-prickle: The effects of positive and negative framing on cooperation in experiments. The Quarterly Journal of Economics, 110, 1-21.

Andreoni, J., \& Miller, J. (2002). Giving according to GARP: An experimental test of the consistency of preferences for altruism. Econometrica, 70, 737-753.

Arrow, K. J., \& Enthoven, A. C. (1961). Quasi-concave programming. Econometrica: Journal of the Econometric Society, 29, 779-800.

Bardsley, N. (2008). Dictator game giving: Altruism or artefact? Experimental Economics, 11, $122-133$.

Cappelen, A. W., Nielsen, U. H., Sørensen, E. Ø., Tungodden, B., \& Tyran, J.-R. (2013). Give and take in dictator games. Economics Letters, 118, 280-283.

Charness, G., \& Rabin, M. (2002). Understanding social preferences with simple tests. Quarterly Journal of Economics, 117, 817-869.

Deaton, A., \& Muellbauer, J. (1980). Economics and consumer behavior. Cambridge University Press.

Eichenberger, R., \& Oberholzer-Gee, F. (1998). Rational moralists: The role of fairness in democratic economic politics. Public Choice, 94, 191-210.

Engel, C. (2011). Dictator games: A meta study. Experimental Economics, 14, 583-610.

Fehr, E., \& Schmidt, K. M. (1999). A theory of fairness, competition, and cooperation. Quarterly Journal of Economics, 114, 817-868.

Fischbacher, U. (2007). z-Tree: Zurich toolbox for ready-made economic experiments. Experimental Economics, 10, 171-178.

Forsythe, R., Horowitz, J. L., Savin, N. E., \& Sefton, M. (1994). Fairness in simple bargaining experiments. Games and Economic Behavior, 6, 347-369.

Greiner, B. (2015). Subject pool recruitment procedures: Organizing experiments with ORSEE. Journal of the Economic Science Association, 1, 114-125.

Jakiela, P. (2013). Equity vs. efficiency vs. self-interest: On the use of dictator games to measure distributional preferences. Experimental Economics, 16, 208-221.

Korenok, O., Millner, E. L., \& Razzolini, L. (2014). Taking, giving, and impure altruism in dictator games. Experimental Economics, 17, 488-500.

Korenok, O., Millner, E. L., \& Razzolini, L. (2018). Taking aversion. Journal of Economic Behavior \& Organization, 150, 397-403.

List, J. A. (2007). On the interpretation of giving in dictator games. Journal of Political Economy, 115, $482-493$.

Moffatt, P. G. (2015). Experimetrics: Econometrics for experimental economics. Macmillan International Higher Education.

Train, K. E. (2009). Discrete choice methods with simulation. Cambridge University Press.

Wales, T. J., \& Woodland, A. D. (1983). Estimation of consumer demand systems with binding nonnegativity constraints. Journal of Econometrics, 21, 263-285.

Zevallos Porles, G. (2018). Essays on antisocial preferences, altruism, and information transmission. Ph.D. thesis, University of East Anglia.

Zizzo, D. (2010). Experimenter demand effects in economic experiments. Experimental Economics, 13, 75-98.

Publisher's Note Springer Nature remains neutral with regard to jurisdictional claims in published maps and institutional affiliations. 